\title{
State of Water in Swollen Membrane in Relation to Permeability
}

\author{
Jiro Komiyama, ${ }^{*}$ Mitsuru Satoh, Wei-Zhong Zhang, \\ akio Nodera, Tomohiro Kusano, Kenzo Ogata, \\ and Shinji TAKaHaSHI
}

Department of Polymer Science, Tokyo Institute of Technology, Ookayama, Meguro-ku, Tokyo 152, Japan

(Received November 26, 1990)

\begin{abstract}
Permeabilities of oxygen and nitrogen gases were measured for crosslinked poly (vinyl alcohol) and poly(4-vinylpyridine) membranes swollen with aqueous solutions of nonvolatile electrolytes and nonelectrolytes. It was found that the permeabilities of the two gases change characteristically with the water content of the membranes, giving the ratio in the range, 25 to 0.5 at around 0.3 of the water content. The importance of the bydration layers of the substrate polymers as the permselective field is suggested based on the measurements of DSC and the partition of the nonvolatile compounds for the swollen membranes. Diffusion selectivity and solubility selectivity comprising the permselectivity are discussed.

KEY WORDS Gas Permeability / Polymer Membrane / Water / Oxygen / Nitrogen / Swelling / Electrolyte / Nonelectrolyte/ Oxygen Enriching Membrane/DSC/
\end{abstract}

Water is a structural liquid. ${ }^{1}$ This should have an influence on the permeability of various gases through the solubility and diffusivity. It is well known that addition of electrolytes and nonelectrolytes to water alters the permeability according to intrinsic properties of the solute molecules. ${ }^{2}$ With aqueous solution, interactions of gassolvent mixture and local hydrodynamics for the gas diffusion may be essential from the elemental consideration of the permeation process. However, aqueous solution is also a structural medium. Alteration of the water structure by the added solute should also have certain effects on the gas permeability. Because of the lack of proper methods to investigate and hence of the experimental observations, such a viewpoint has never been proved to have reality and has remained as a mere speculation. The present report summarizes our study on the extraordinary permselectivities for oxygen over nitrogen, or nitrogen over oxygen, observed for membrane systems composed of hydrophilic polymer/water/ nonvolatile additives. The basic idea for constructing such systems is that nearby coexistence of polymers and additives, both of which have specific hydrations around themselves, may provide an effective field for selective gas permeation. The polymers so far investigated are 
poly (vinyl alcohol) (PVA) and poly(4-vinylpyridine) (P4VP) and the add $\mathrm{i}$ tives are over 30 compounds including metal salts, ureas, polyols and sugars. With certain additives which were sorbed in the membranes as 10 or 20 weight $\%$ aqueous solution, the permeability ratios of oxygen over nitrogen were found to be 25 to ca. 0.5 , i. e., highly permselective for oxygen or so for nitrogen. Such selective permeations were observed for the membranes with the water content, $H$ in the weight fraction, less than 0.50, and indeed the selectivity became higher as the content became smaller to ca. 0.30. Although our study is lead by the aformentioned viewpoint, the present stage of the investigation is not such as to present here any established interpretation but to present only the highly permselective phenomena observed for more than several ternary systems. These may be interesting, however, as novel permselective systems awaiting physico-chemical understanding.

\section{EXPER IMENTAL}

PVA membranes crosslinked with glutaraldehyde to different degrees were prepared by the conventional method. ${ }^{3}$ The crosslinking was adjusted to make the water contents of the membranes in 0.25-0.80. P4VP membranes crosslinked with 1,6-dibromohexane to different degrees were prepared. ${ }^{4}$ Due to the low hydrophilicity of the membrane, the water contents were adjusted to $0.28-0.35$. These membranes were soaked in the 10-20 weight $\%$ aqueous solutions of nonvolatile compounds at room temperature for 3 days. The membranes were wiped with filter paper, and then set in the cell of gas permeation measurement equipment. ${ }^{5}$ The measurements were done at $26^{\circ} \mathrm{C}$ under equilibrium water vapor pressure, usually under ca. 1 atm $/ 0$ atm pressure difference of oxygen or nitrogen gases separately. By adjusting the membrane thicknesses appropriately, the permeation curves were followed within 10-240 minutes. Conventional analyses of the curves gave the permeability from the linear part and the apparent diffusion coefficient by the extraporation. Partition of the added compounds in the membrane from the aqueous solution were measured by the desorption method. Differential scanning calorimetry of the solution swollen membranes was performed with a Seiko DSC-10 calorimeter. ${ }^{6}$

\section{RESULTS AND DISCUSSION}

The permeation of oxygen and nitrogen gases were measured for the PVA membrnes swollen $w$ ith aqueous solutions of 28 compounds 1 is ted in Table 1. The concentrations of the compounds were $0-20$ weight $\%$. As shown by the thick lines in Fig. 1, PVA membranes swollen with water gives a permeability ratio, 2.5, for oxygen over nitrogen, regardless of the water content of the membrane, 0.25-0.80. Among alkali chlorides, namely $\mathrm{LiCl}, \mathrm{NaCl}, \mathrm{KCl}$ and $\mathrm{CsCl}, \mathrm{LiCl}$ was found to be the most effective for the permselectivity. Fig. 1 shows the permeabilities of oxygen and nitrogen against the water content of the membranes swollen with $10 \%$ 
LiCl solution. This is a typical example of the permeability variations to give high permselectivity for oxygen;oxygen permeability is almost the same or slightly lower than those observed ith the water swollen membranes, while nitrogen permeability is greatly suppressed at the low water content region. $\mathrm{NaCl}$ gives the same tendencies in the permeability variations but to far less extents than LiCl. KCl and $\mathrm{CsCl}$ give no deviation from the water swollen membranes.

Among $d i-$ and $t r i-v a l e n t$ metal chlorides, $\mathrm{MgCl}_{2}, \mathrm{CaCl}_{2}, \mathrm{BaCl}_{2}$, $\mathrm{ZnCl}_{2}$ and $\mathrm{AlCl}_{3}, \mathrm{MgCl}_{2}$ is the most effective for the selective permeation. Fig. 2 shows again that the nitrogen permeabilities lowered at the membrane water content less than ca. 0.50 while the oxygen permeability is persistent. $\mathrm{CaCl}_{2}, \mathrm{ZnCl}_{2}$ and $\mathrm{AlCl}_{3}$ give the same tendencies but to lesser extents. $\mathrm{BaCl}_{2}$ has no effect at all. These observations show that the effective metal salts, lithium, magnesium and calcium chloride, are those composed of metal cations having relatively small ion size. This may be restated as follows; metal cations which are strongly hydrated are effective

Table I Compounds used for the solution swollen PVA membranes*

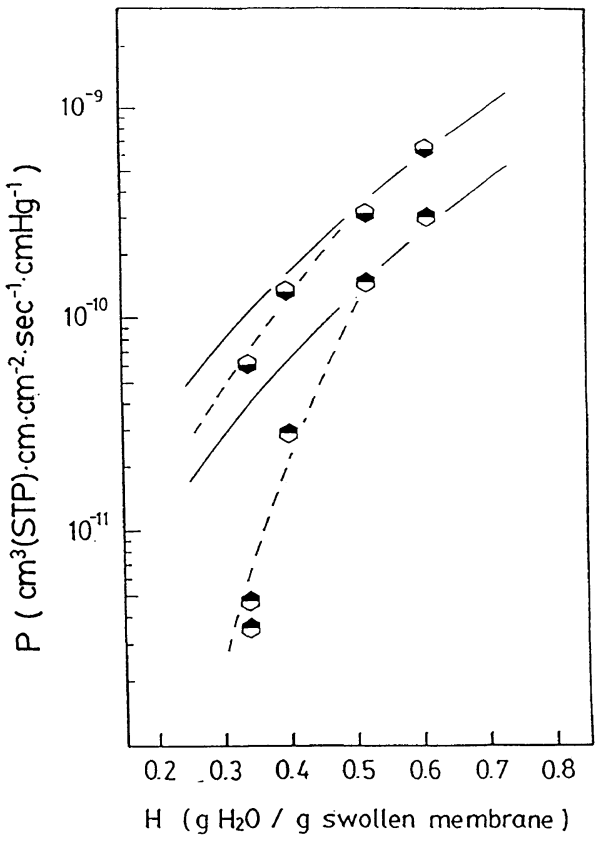

Figure 1. Permeabilities to (O) $\mathrm{O}_{2}$ and $(\Theta) \mathrm{N}_{2}$ of PVA membranes swollen with $10 \%$ aq. LiCl. Thick lines represent the values of water swollen membranes.

\footnotetext{
Electrolytes

$\mathrm{LiCl}(17)^{\cdots} \quad \mathrm{NaCl}(4.0) \quad \mathrm{KCl} \quad \mathrm{CsCl} \quad \mathrm{LiBr} \quad \mathrm{LiI}(15) \quad \mathrm{LiNO}_{3}$

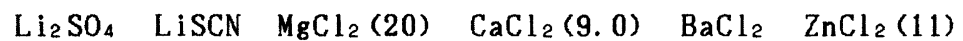

$\mathrm{AlCl}_{3}(6.6) \quad$ (Et) ${ }_{4} \mathrm{NCl} \quad \mathrm{NaB}(\mathrm{ph})_{4} \quad \mathrm{CH}_{3}\left(\mathrm{CH}_{2}\right)_{15} \mathrm{~N}\left(\mathrm{CH}_{3}\right)_{3} \mathrm{Br}$

Li $\beta$-naph thal enesulphona te (25)

Nonelectrolytes

urea (6.1) thiourea(4.0) glycerol (0.5) $\cdots$

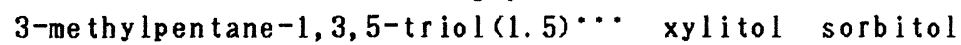

glucose sucrose raffinose

None

water (2.5)

* These compounds were used as 5,10 or 20 weight $\%$ solutions.

** The numbers in the parentheses represent the highest $\mathrm{O}_{2} / \mathrm{N}_{2}$ permselectivity observed for the relevant membrane system.

*** The numbers represent the lowest $\mathrm{O}_{2} / \mathrm{N}_{2}$ permselectivity.
} 
for the present purpose. Finding these facts, one might be interested in the effect of the anion species on the permselectivity. This was studied with lithium salts of chloride, bromide, iodide, nitrate, thiocyanate, sulfonate and $\beta$-naphthalenesulfonate. The selection of these salts was made rather arbitrarily. But, for chloride, iodide and naphthalenesulfonate, remarkable oxygen permselectivity, 17-25, caused by the suppression of nitrogen permeability, was found. Fig. 3 shows the naphthalenesulfonate case. Compared with these salts, the other salts give smaller suppressions of nitrogen permeability as well as some suppressions of oxygen permeability. Hence the permselectivities are not so high. These effects of the anions do not seem simply to be correlated with any property of the anions, e. g., the hydration nature like structure forming or structure breaking. This may be natural since the hydrations of anions are much weaker than cations.

Salts are sorbed by the water swollen membrane where they work effectively to modify the gas permeability. If we define the partition coefficient by the concentration based on the water in the membrane divided by that in the outer solution, it is expected to be lower than $1 \mathrm{be}^{-}$ cause of the low dielectric constant of the membrane substrate. This is not the case for PVA membranes swollen with the solutions of the effective salts, as found in the examples shown in Fig. 4. Among the partition coefficients measured for the metal chlorides mentioned above, those of $\mathrm{CsCl}$ and $\mathrm{BaCl}_{2}$ show marked decreases to $0.5-0.6$ with the decrease in the membrane water content to 0.30. The other salts were found to keep the values higher than or equal to ca. $1( \pm 10 \%)$

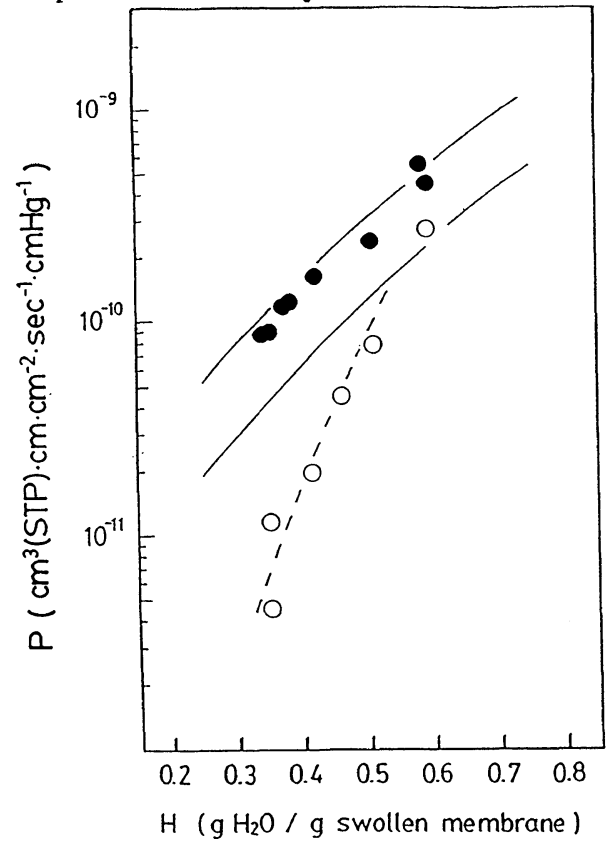

Figure 2. Permeabilities to (O) $\mathrm{O}_{2}$ and (O) $\mathrm{N}_{2}$ of PVA membranes swollen with $10 \%$ aq. $\mathrm{MgCl}_{2}$.

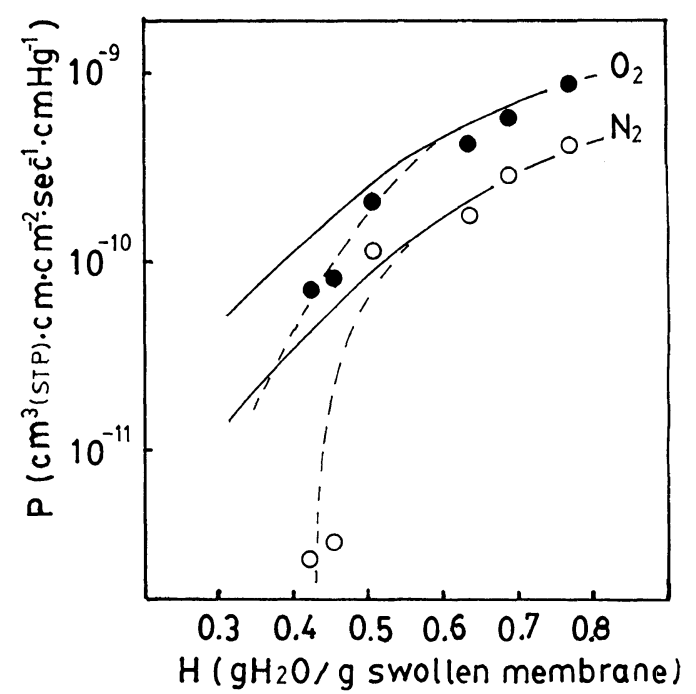

Figure 3. Permeabilities to (O) $\mathrm{O}_{2}$ and $(\mathrm{O}) \mathrm{N}_{2}$ of PVA membranes swollen with $5 \%$ aq. Li $\beta$-naphthalenesulfonate. 
regardless of the water content. These observations seem to imply that the presence of appropriate amount of salts very close to PVA chain or in the hydration layer of the polymer is a prerequis ite to form the permselective field for oxygen or nitrogen. The high affinities to PVA found for the effective salts may be of various origins; interactions between the polymer and the hydrophobic moieties of naphthalenesulphonate, hydrogen bonding between the polymer and glycerol and affinity to the polymer hydration layer, we suggest, of lithium chloride and iodide. This argument gives us a reason to search for the wide variety of nonvolatile compounds of different natures.

Although not many nonelectrolytes have been tested as additives for PVA membranes, some of which show characteristic effects for the permselectivity. Urea, when added to solution up to $2.5 \%$, enhanced the permeabilities of both oxygen and nitrogen to the membranes with $H$ less than 0.50 , resulting in the permeability ratio, 6. 1 at $\mathrm{H}=0.40 .{ }^{3} \quad$ Thiourea was found to be not so much effective. ${ }^{6}$ Glycerol, when used as $20 \%$ aqueous solution, gives an inversion of the oxygen and nitrogen permeabilities at $\mathrm{H}=0.30$. Following this, 3-me thylpentane-1, 3,5triol, xylitol, sorbitol, glucose, sucrose and raffinose were tested as additives.

Fig. 5 shows the glycerol case, which anyhow indicates that oxygen permeability deviates downward to apprioach that of nitrogen at the lowest water content region. The same

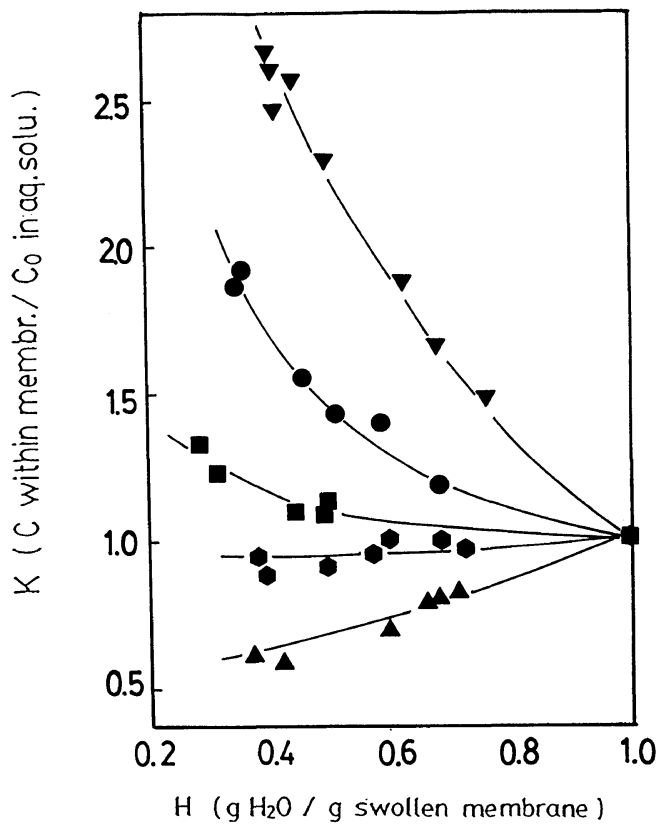

Figure 4. Partition coefficients of ( $\nabla)$ Li $\beta$-naphthalenesulfonate, (O) $\mathrm{LiI},(\mathbb{\square})$ glycerol, (O) $\mathrm{LiCl}$ and ( $\boldsymbol{\Delta}) \mathrm{CsCl}$.

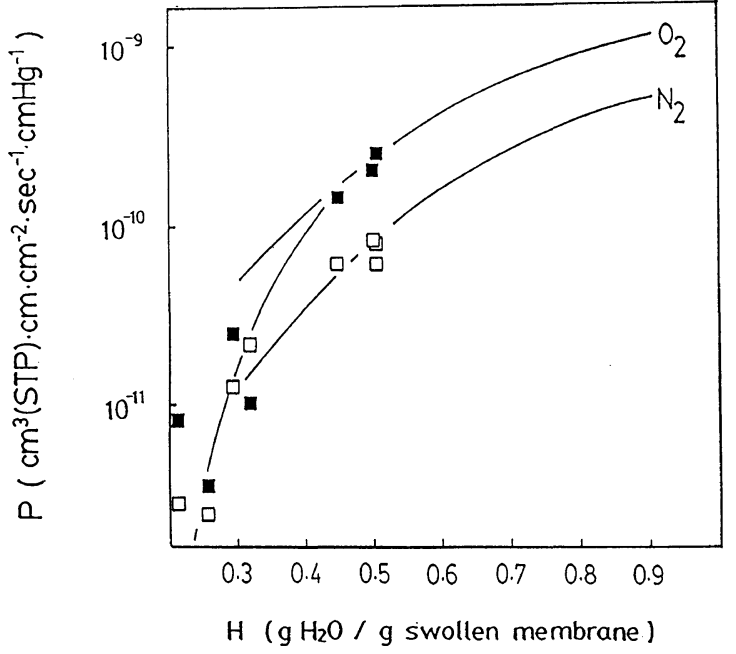

Figure 5. Permeabilities of ( $\square$ ) $\mathrm{O}_{2}$ and ( $\square$ ) $\mathrm{N}_{2}$ to PVA membranes swollen with $20 \%$ aq. glycerol. 
trend of the two gas permeabilities were observed for the pentane-triol and sucrose cases. These results suggest that the ternary membrane systems may work as nitrogen permselective one, according to the nature of the compounds added. Further study is now in progress.

Permeability coefficient of a multi phase membrane is given by $P=\sum D_{i} S_{i}$

where $D_{i}$ and $S_{i}$ stand for the diffusion coefficient and solubility coefficient in i phase, respectively. For the present membrane, the whole system may be divided into three phases, polymer, the hydration layer containing the added solute and the bulk solution inside. This classification is supported by the observation that nearly constant amount of so called nonfreezing water and increasing amount of freezing water per unit weight of the polymer are detected for $H$ larger than 0.4 by DCS measurements. ${ }^{7}$ Then, the diffusion coefficient estimated from the extrapolation of the linear part of the permeation-curve becomes ${ }^{8}$ $D=\sum D_{i} S_{i} / \Sigma S_{i}$

and the solubility coefficient, $S$, calculated by $P / D$ is $S=\Sigma S_{i}$

Note that such a formulation holds for the case that gas dissolution in each phase is of partition type. In relation to this, the dependences of the permeabilities on the upstrem pressure, 0.3-3.2 atm, were measured. Fig. 6 shows an example, where we find constant permeabilities of oxygen and nitrogen for the membrane swollen with $5 \%$ aqueous LiCl to $H=0.36$. Such constancy holds also for the membranes swollen with 10 $\%$ aqueous $\mathrm{MgCl}_{2}$ and $\mathrm{CaCl}_{2}$. The observed $\mathrm{D}$ and calculated $\mathrm{S}$ for LiCl, LiI and Li $\beta$-naphthalenesulphonate systems are shown in Figs. 7 and 8 against $H$. The data are scattered but in both figures, different variations of the two coefficients for oxygen and nitrogen may be perceived. With the decrease in $H$, both of the diffusion coefficients of oxygen and nitrogen becomes small from the very nature of the relevant systems, but the ratio becomes high for $H$ smaller than 0.4. This means that diffusion selectivity for the two gases appears at the low water content region. Also the solubilities of the two gases show different variations with $H$. It is perceived again that $S$ of oxygen becomes noticeably high at H smaller than 0.4, while those of nitrogen remain at a certain level. This means that solubility selectivity also appears for the relevant systems at the low water content region. Thus, the high permselectivities found for these systems are derived from the two factors. It is quite natural to

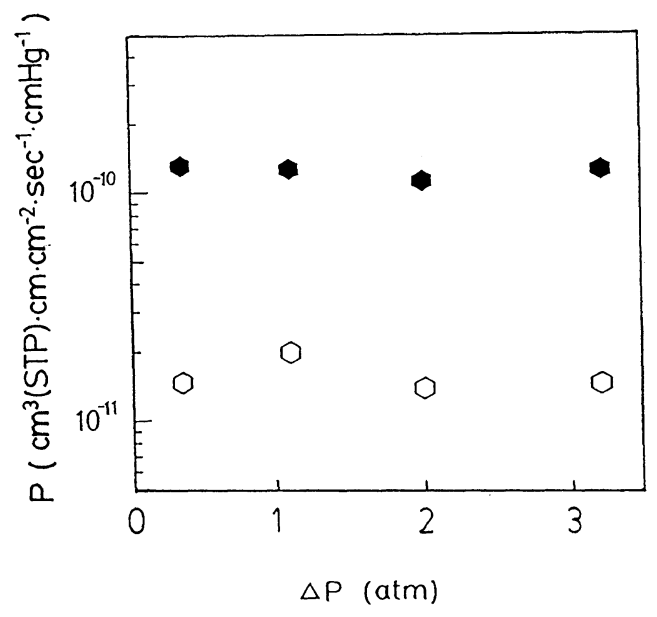

Figure 6. Dependence on upstream pressure of permeabilities of $\mathrm{O}_{2}$ and $(O) \mathrm{N}_{2}$ to PVA membrane swollen with $5 \%$ aq. LiCl. 
assign the polymer hydration layer to the phase effective for such characteristic behaviors. If we assume the variations of $D$ and $S$ of nitrogen with $\mathrm{H}$ to be standard, it is said that the bydration layer has an extra solubilizability to oxygen of which diffusivities are not much suppressed or even enhanced. Because of the scatter of the data points, it may be dangerous to advance the discussion along these lines. However, the greatest reason for the scatter lies in the preparative method of the meinbranes with different water contents. For a single membrane, oxygen and nitrogen permeations were repeatedly measured. This implies that the ratios of $D$ and $S$ for the two gases are more reliable for the discussion. Fig. 9 shows schematically the ratios agains $\mathrm{H}$ for the three types of characteris$t$ ic variations found for the PVA systems. The first one, which includes LiCl, LiBr, LiI and Li $\beta$ naphthalenesulfonate systems, is the type just discussed above. At low H, both ratios becomes high, resulting in the high permselectivity for oxygen. The second case, which includes $\mathrm{Li}_{2} \mathrm{SO}_{4}$ and $\mathrm{LiNO}_{3}$, shows inverse variations of the ratios with $H$. At low $H$, the ratio of $S$ becomes high but the decrease in the ratio of D compensates it, which results in the almost invariant permselectivity for oxygen over nitrogen over the whole range of H. The third case, which includes glycerol, pentane-triol and sugars, again show inverse variations of the two ratios with H. The solubility ratio becomes lower than 1 with the decrese in $H$. Respective solubility data indicate that this variation is brought about by the in-

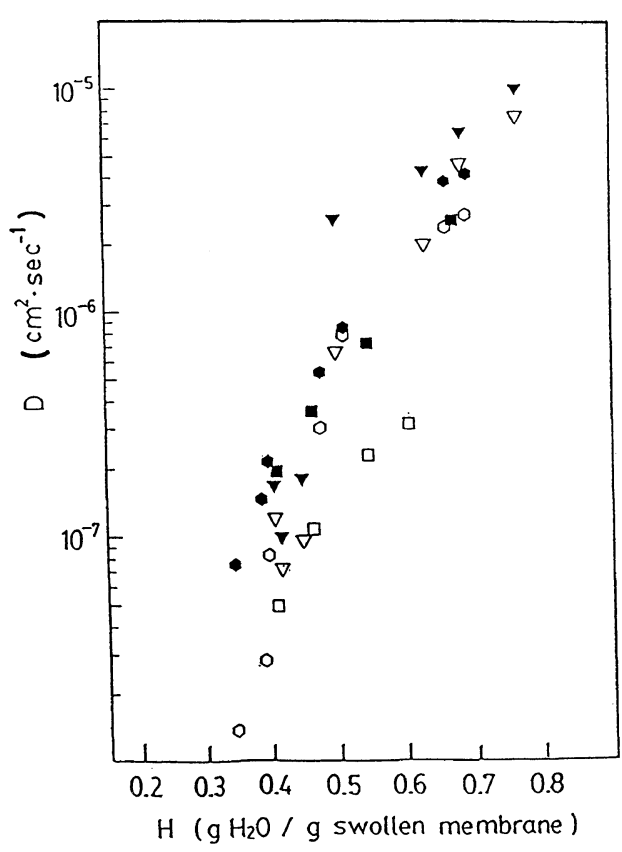

Figure 7. Diffusion coefficients of $\mathrm{O}_{2}$ (filled) and $\mathrm{N}_{2}$ (open) for PVA membranes swollen with $(O, O) 10$ $\%$ aq. $\mathrm{LiCl},(\square, \square) 10 \%$ aq. LiI and $(\nabla, \nabla) 10 \%$ aq. Li $\beta$-naphthalenesulfonate.

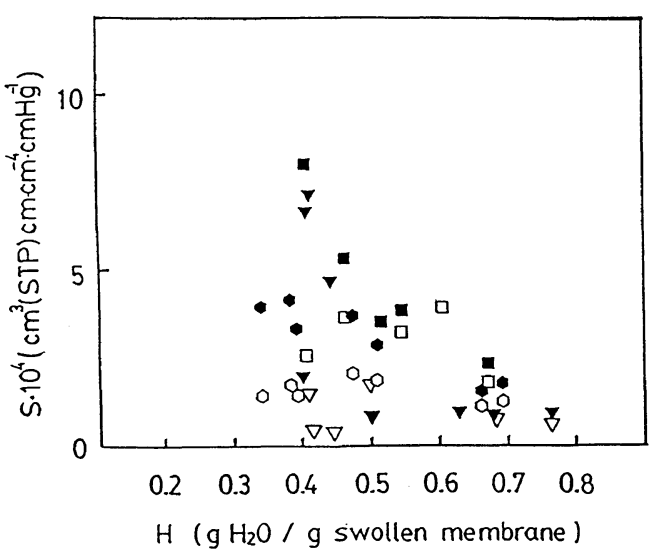

Figure 8. Solubility coefficients of $\mathrm{O}_{2}$ and $\mathrm{N}_{2}$ for PVA membranes swollen with $10 \%$ aq. salts. See Fig. 7 for the symbols. 
crease in the solubility of nitrogen at low $H$. On the other hand, the diffusion $\mathrm{co}^{-}$ efficient ratio keeps the value of around 1 until $\mathrm{H}$ approaches to 0.3 and then it rises to high values over 10 . This variation is brought about by the sharp decrease in the diffusion $\mathrm{co}^{-}$ efficient of nitrogen at low $H$. It is near the critical H at around 0.3 that the low solubility ratio dominates the permselectivity to be smaller than 1 , i. e., nitrogen becomes permselective. With further decrease in $H$, the membranes become again oxygen permselective due to the rise of the $D$ ratio. With reservations on the insufficient reliability of the present data, we conclude that the selective solubilization of the two gases is accompanied $e$ ither by the increase or by the decrease of the diffusivity, according to the nature of the added compounds. We stress here again that it is the hydration layer which works at the permselective field.

Permselective PVA membrane systems have been discussed so far. Once the findings being confirmed, it may be important to see if such a permselective system is confined to PVA membrane or not. Poly (4-vinylpyridine) was selected as the second membrane substrate only for the reason that the membrane was easily available in our laboratory. The interesting point for this membrane may be found in the coordination of the pyridine groups to metal cations such as nickel and cobalt. The permeabilities of oxygen and nitrogen were measured for the membranes swollen with aqueous solutions of the chlorides of $\mathrm{Ni}, \mathrm{Co}, \mathrm{Mg}$ and $\mathrm{Li}$. The latter two salts were studied to find the effects of noncoordinating metal salts. Fig. 10 shows the permeabilities of the two gases to the solution swollen membranes together with those swollen with water. It is seen that all of the metal chlorides enhance the permselectivities to the values larger than 5 which is definitely larger than the value, 2.7, observed for the water swollen membranes. These results indicate that, aside from the vast possibilities in the selection of the nonvolatile additives, the hydrophilic polymer substrate is also the subject for a wide selection. In this context, we should be aware of the 
fact that not much knowledge has been accumulated for the interaction of the hydrophilic polymers with electrolytes and nonelectrolytes.

Finally we summarize the facts we found, their interpretations, and the implications. For PVA/water /nonvolatile compound and poly (4-vinylpyridine)/water /metal chloride membrane systems, permselectivities in the range 25-0.5 for oxygen were found. Representative permselectivities are plotted against the permeability of oxygen in Fig. 11. The effective compounds, although literally they may be only some broken pieces of a floating iceberg, include electrolytes and nonelectrolytes. The partition data for the additives suggest that effective compounds have affinity to the membrane composing polymer and/or to the bydration layer through various interactions. From the diffusion and solubility coefficients of the gases, it is discussed that extra-solubilization of either gas takes place in the hydration layer of the polymer and is accompanied either by the increase or by the decrease of the diffusivity. Permselectivities found for the two polymer membrane systems encourage us to advance the search for a bighly effective hydrophilic polymer membrane.

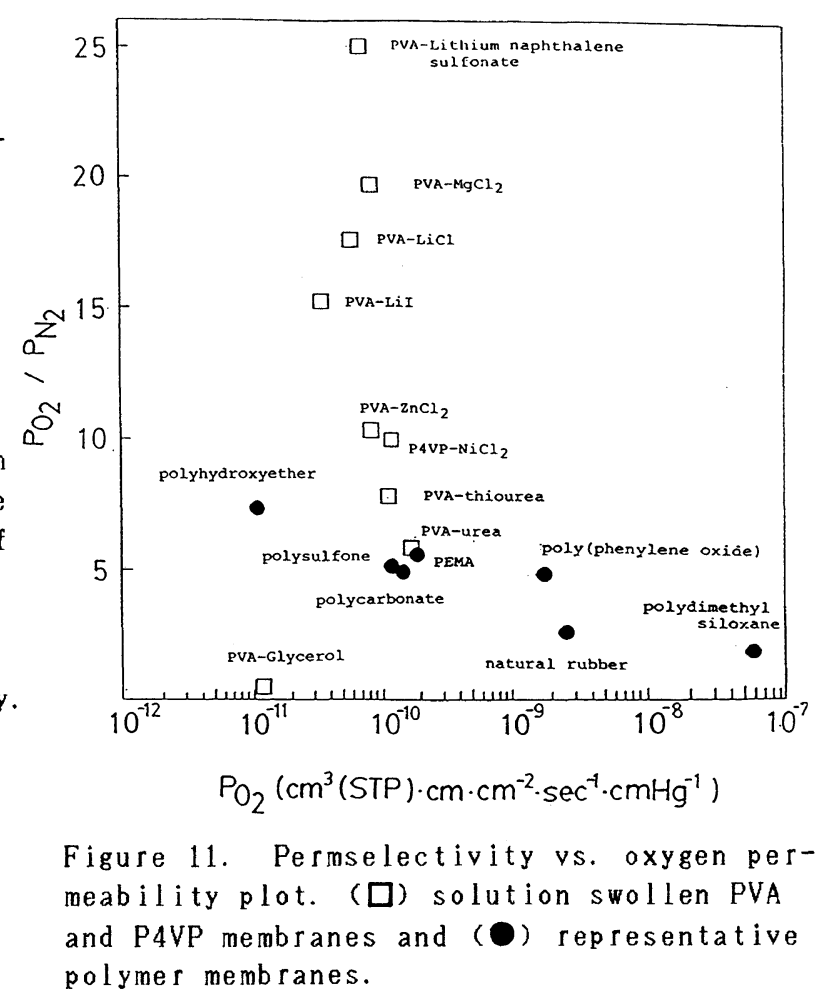




\section{ACKNOWLEDGEMENT}

This work was partially supported by a Grant-in-Aid for Scientific Research (No.61550653) from the Ministry of Education, Science and Culture of Japan.

\section{REFERENCES}

1. "Water A Comprebensive Treatise" ed. F. Franks, vol. 1, Prenum, N. Y. -London, 1972.

2. ibid, vol. 2, 3 and 4, 1973, 1974 and 1975.

3. W. -Z. Zhang, M. Satoh and J. Komiyama, J. Membrane Sci., 31, 147 (1987).

4. K. Ogata, M. Satob and J. Komiyama, presented at the 3rd International Polymer Conference (IPC 90), Nagoya, November 26-29, 1990.

5. W. - Z. Zhang, M. Satoh and J. Komiyama, J. Membrane Sci., 40, 343 (1989).

6. W. -Z. Zhang, M. Satoh and J. Komiyama, J. Membrane Sci., 35, 311 (1988).

7. W. - Z. Zhang, M. Satoh and J. Komiyama, J. Membrane Sci., 42, 303 (1989).

8. A. Higuchi, H. Fushimi and T. Iijima, J. Membrane Sci., 25, 171 (1985). 